\title{
Association Between Duration of Dysphagia Recovery and Lesion Location on Magnetic Resonance Imaging in Patients With Middle Cerebral Artery Infarction
}

\author{
Jae Ho Kim, MD, Se Hyun Oh, MD, Ho Joong Jeong, MD, PhD, \\ Young Joo Sim, MD, PhD, Dung Gyu Kim, MD, Ghi Chan Kim, MD, PhD
}

Department of Physical Medicine and Rehabilitation, Kosin University Gospel Hospital, Busan, Korea

Objective To investigate association between lesion location on magnetic resonance imaging (MRI) performed after an infarction and the duration of dysphagia in middle cerebral artery (MCA) infarction.

Methods A videofluoroscopic swallowing study was performed for 59 patients with dysphagia who were diagnosed as cerebral infarction of the MCA territory confirmed by brain MRI. Lesions were divided into 11 regions of interest: primary somatosensory cortex, primary motor cortex, supplementary motor cortex, anterior cingulate cortex, orbitofrontal cortex, parieto-occipital cortex, insular cortex, posterior limb of the internal capsule (PLIC), thalamus, basal ganglia (caudate nucleus), and basal ganglia (putamen). Recovery time was defined as the period from the first day of L-tube feeding to the day that rice porridge with thickening agent was prescribed. Recovery time and brain lesion patterns were compared and analyzed.

Results The mean recovery time of all patients was $26.71 \pm 16.39$ days. The mean recovery time was $36.65 \pm 15.83$ days in patients with PLIC lesions and 32.6 \pm 17.27 days in patients with caudate nucleus lesions. Only these two groups showed longer recovery time than the average recovery time for all patients. One-way analysis of variance for recovery time showed significant differences between patients with and without lesions in PLIC and caudate $(\mathrm{p}<0.001)$.

Conclusion Injury to both PLIC and caudate nucleus is associated with longer recovery time from dysphagia.

Keywords Dysphagia, Middle cerebral artery, Infarction

Received April 30, 2018; Accepted October 8, 2018

Corresponding author: Ghi Chan Kim

Department of Physical Medicine and Rehabilitation, Kosin University Gospel Hospital, 262 Gamcheon-ro, Seo-gu, Busan 49267, Korea. Tel: +82-51990-6156, Fax: +82-51-241-2019, E-mail: ghichan@hotmail.com

ORCID: Jae Ho Kim (http://orcid.org/0000-0002-3556-4194); Se Hyun Oh (http://orcid.org/0000-0001-6754-8669); Ho Joong Jeong (http://orcid. org/0000-0002-0607-2799); Young Joo Sim (http://orcid.org/0000-0002-0640-8766); Dung Gyu Kim (http://orcid.org/0000-0002-9484-6562).; Ghi Chan Kim (http://orcid.org/0000-0002-4954-3175).

(a) This is an open-access article distributed under the terms of the Creative Commons Attribution Non-Commercial License (http://creativecommons.org/ licenses/by-nc/4.0) which permits unrestricted noncommercial use, distribution, and reproduction in any medium, provided the original work is properly cited. Copyright $\odot 2019$ by Korean Academy of Rehabilitation Medicine 


\section{INTRODUCTION}

Swallowing disorders occur in about $30 \%-50 \%$ of patients who have survived stroke. They are risk factors for aspiration pneumonia, malnutrition, and dehydration [1]. They also increase the length of hospital stay, interfere with rehabilitation, and increase the risk of death in severe cases. The risk of aspiration pneumonia in a stroke patient with dysphagia is three times higher than that in a stroke patient without dysphagia. In addition, the mortality rate is three times higher in stroke patients with pneumonia $[2,3]$. Therefore, early detection and treatment of swallowing disorders should be a priority in rehabilitation of stroke patients to reduce morbidity, malnutrition, and the risk of death. Before the first oral intake of fluids or food after a stroke, a dysphagia test is recommended regardless of stroke severity. Clinical guidelines based on systematic reviews should be used $[1,2]$.

However, unlike dysarthria or aphasia that can be quickly diagnosed as early symptoms of stroke, symptoms of dysphagia are less prominent. Thus, evaluation and treatment of dysphagia may be delayed [4]. Therefore, magnetic resonance imaging (MRI)-based predictors of swallowing disorders are useful for early management. Several studies have attempted to identify lesion patterns that can predict swallowing disorders. However, these failed to yield meaningful results due to small sample sizes and roughly classified brain regions $[5,6]$. Recent, larger studies that overcame these limitations examined effects of lesion location on dysphagia incidence, pattern, and complications in acute stroke [7-9]. However, most studies were focused on the incidence of dysphagia. Reports on the association between lesion location and duration of dysphagia recovery are lacking [10]. Because the occurrence of dysphagia is associated with various swallowing mechanisms, reported parameters have limitations to identify primary brain lesion in dysphagia. We hypothesized that primary brain lesion location could be associated with severity of dysphagia or time to recovery. Thus, the aim of this study was to investigate the association between brain lesion location on MRI performed after infarction and the duration of dysphagia recovery in patients with middle cerebral artery (MCA) infarction.

\section{MATERIALS AND METHODS}

\section{Subjects}

This retrospective study included 59 stroke patients treated at the rehabilitation department of Kosin University Hospital between 2012 and 2016. Patient inclusion criteria were as follows: (1) cerebral infarction in the MCA territory, (2) L-tube feeding for dysphagia, (3) follow-up brain MRI within one week of stroke onset, (4) treatment for dysphagia after videofluoroscopic swallowing study (VFSS), and (5) L-tube feeding after initial VFSS. Exclusion criteria were as follows: (1) cerebral infarction involving sites other than those in MCA territory, (2) previous cerebral infarction, (3) bilateral hemisphere lesions, (4) lack of follow-up VFSSs, and (5) interruption of dysphagia therapy. Fifty-nine out of 162 stroke patients who met these inclusion criteria were finally enrolled for this study (Table 1). The Institutional Review Board approval was granted by Kosin University College of Medicine (IRB No. 201404-BM-005).

\section{Dysphagia assessment}

VFSS was performed by an experienced rehabilitation physician who was assisted by a radiologic technician. The subject swallowed a bolus containing mixed barium powder. A 2- or 5-mL bolus consisting of pudding,

Table 1. General characteristics of subjects $(n=59)$

\begin{tabular}{|lc|}
\hline \multicolumn{1}{c}{ Characteristic } & Value \\
\hline Age $(\mathrm{yr})$ & $65.84 \pm 10.1$ \\
Sex, male & $30(50.8)$ \\
\hline Cause of ischemia & \\
\hline Cardioembolic & $25(42.4)$ \\
\hline Large artery & $21(35.6)$ \\
\hline Other & $13(22.0)$ \\
\hline Thrombolysis & $15(25.4)$ \\
\hline mRS & $3.1 \pm 1.5$ \\
\hline NIHSS & $7.7 \pm 6.5$ \\
\hline Time to first VFSS (day) & $13.6 \pm 7.7$ \\
\hline Recovery time to & $26.71 \pm 16.39$ \\
\hline dysphagia diet stage 3 (day) & \\
\hline
\end{tabular}

Values are presented as mean \pm standard deviation or number (\%).

mRS, Modified Rankin Scale; NIHSS, National Institutes of Health Stroke Scale; VFSS, videofluoroscopic swallowing study. 
mashed banana, porridge, rice, and water was used. The test was stopped if aspiration occurred or residual volume was high. Subjects were evaluated for lip closure, bolus formation, residue in the oral cavity, oral transit time, laryngeal elevation and epiglottic closure, nasal penetration, residue in the valleculae, residue in the pyriform sinuses, coating of the pharyngeal wall after swallowing, and pharyngeal transit time. VFSS was performed at intervals of one week after the first evaluation.

Diet

After evaluation, patients were prescribed a diet according to VFSS results. For a patient to reach the third stage of a dysphagia diet, the following conditions must have been met: (1) intact lip closure, (2) no aspiration of a solid diet such as pudding or crushed banana, (3) grade 1 or less residual volume in the oral cavity, valleculae, and pyriform sinuses after double swallowing, (4) no significant delay in oral or pharyngeal transit time, and (5) normal laryngeal elevation and epiglottic closure.

\section{Dysphagia therapy}

Dysphagia therapy was performed in patients with swallowing disorders identified using VFSS. Three occupational therapists performed dysphagia therapy five times a week (60 minutes each time). Dysphagia therapy included vital stimulation of the submental region, anterior belly of the digastric muscle, thyroid muscle, and middle pharyngeal constrictor muscle by attaching channels between the jaw tip and hyoid bone. Oromotor training including soft plate stimulation, tongue massage, and ice stick stimulation was also performed.

\section{Findings on MRI}

Clearly defined ischemic lesions were evaluated on follow-up MRI 24-72 hours after symptom onset. The radiologist was blinded to patient swallowing function.

On MRI, brain lesions locations were divided into primary somatosensory cortex, primary motor cortex, supplementary motor cortex, anterior cingulate cortex, orbitofrontal cortex, parieto-occipital cortex, insular cortex, posterior limb of the internal capsule (PLIC), thalamus, basal ganglia (caudate nucleus), and basal ganglia (putamen) [11].

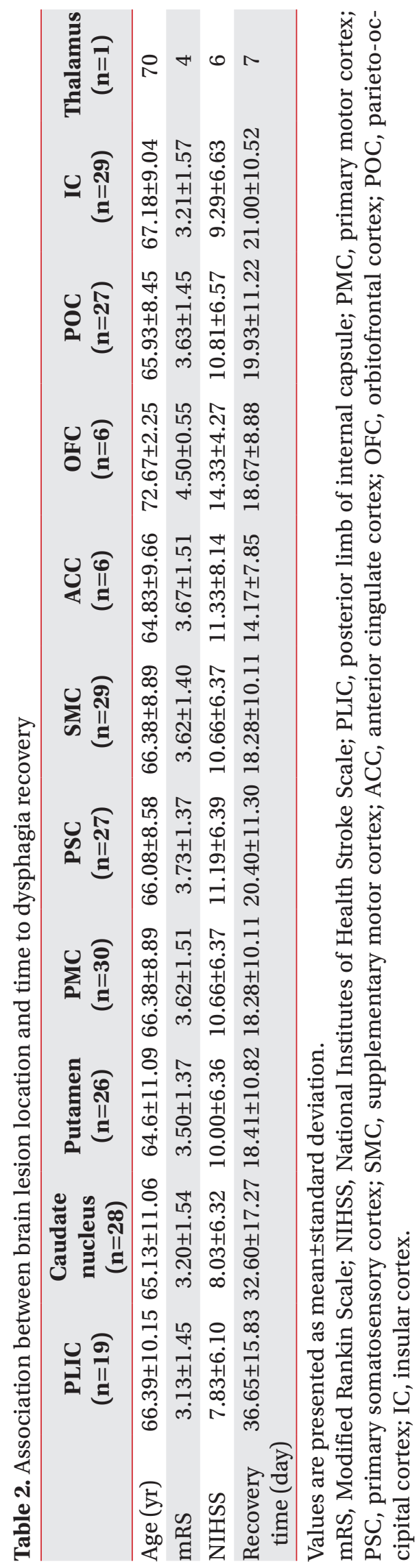




\section{Data analysis}

SPSS for Windows 20.0 (IBM, Armonk, NY, USA) was used for all statistical analyses. Patients receiving L-tube feeding at the first VFSS underwent dysphagia therapy. Recovery time was the period from the first VFSS at which L-tube feeding was prescribed to the VFSS at which rice porridge with thickening agent was prescribed. Age, Modified Rankin Scale (mRS) score, National Institutes of Health Stroke Scale (NIHSS) score, and recovery time in all patients and lesion-classified groups were analyzed. We compared groups with longer recovery time to those with average recovery time. Lesions showing significant results were further classified according to whether lesions overlapped. One-way analysis of variance (ANOVA) was used to examine differences in recovery time between groups. Independent samples t-test was used to compare recovery responses according to the presence or absence of a lesion. Chi-square test was used to compare general patient characteristics. A p-value of less than 0.05 was considered significant.

\section{RESULTS}

Mean age, mRS score, NIHSS score, and recovery time for all patients were $65.84 \pm 10.1$ years, $3.1 \pm 1.5,7.7 \pm 6.5$, and $26.71 \pm 16.39$ days, respectively. Dysphagia recovery time was longer in patients with lesions of the PLIC or caudate nucleus (Table 2). The average recovery time was $36.65 \pm 15.83$ days in patients with PLIC lesions and $32.6 \pm 17.27$ days in patients with caudate nucleus lesions. The mean recovery time in patients without lesions of both PLIC and caudate nucleus, those with a lesion of the PLIC only, those with a lesion of the caudate nucleus only, and those with lesions of both the PLIC and caudate nucleus were $12.9 \pm 6.7,24.7 \pm 14.0,24.6 \pm 22.9$, and
39.4 \pm 20.0 days, respectively (Table 3 ). One-way ANOVA showed statistically significant differences in mean recovery time between groups $(\mathrm{p}<0.001)$. Scheffe multiple comparison test showed significant differences between patients with and without lesions in PLIC and caudate $(\mathrm{p}<0.001)$ (Table 3).

\section{DISCUSSION}

Various studies have attempted to identify the location of the swallowing center. Recently, the right cerebral hemisphere has been reported to have a greater effect on swallowing function than the left cerebral hemisphere $[7,12]$. However, swallowing requires the interaction of both cerebral hemispheres. Left hemisphere lesions are associated with oral phase dysfunction while right hemisphere lesions are associated with pharyngeal phase impairment $[13,14]$. Studies on voluntary and reflexive swallowing centers have shown various findings. Kern et al. [15] have reported that swallowing centers are in the bilateral primary motor and sensory regions, insular cortex, and prefrontal lobe. Flowers et al. [16] have reported that pontine and medullary lesions are associated with dysphagia. Some studies have suggested that internal capsular lesions are associated with dysphagia $[17,18]$. These results suggest that the bilateral neural network from the medulla to cerebral hemispheres mediates swallowing.

The internal capsule is a pathway for large thalamocortical, corticothalamic, corticopontine, corticobulbar, and corticospinal fibers. The PLIC lies between the lenticular nucleus and thalamus. It is the pathway for corticospinal and corticobulbar tracts originated in the motor cortex, ventral posterolateral nucleus, and ventral posteromedial. The corticobulbar tract travels from the ventral mo-

Table 3. Recovery time by lesion of PLIC or caudate nucleus

\begin{tabular}{lccccc}
\hline & Group A (n=24) & Group B (n=7) & Group C (n=16) & Group D (n=12) & p-value \\
\hline Recovery time (day) & $12.9 \pm 6.7$ & $24.7 \pm 14.0$ & $24.6 \pm 22.9$ & $39.4 \pm 20.0$ & $0.001^{* * *}$ \\
\hline $\mathrm{T}^{\mathrm{a})}$ & $\mathrm{a}$ & $\mathrm{a}, \mathrm{b}$ & $\mathrm{a}, \mathrm{b}$ & $\mathrm{b}$ & \\
\hline
\end{tabular}

Values are presented as mean \pm standard deviation.

Groups were divided according to overlap of posterior limb of internal capsule (PLIC) and caudate nucleus lesions: group A, patients without a lesion of both the PLIC caudate nucleus; group B, patients with a lesion of the PLIC only; group C, patients with a lesion of the caudate nucleus only; group D, patients with lesions of both PLIC and caudate nucleus.

${ }^{a)}$ The same letters indicate non-significant difference between groups based on Scheffe multiple comparison test. *** $\mathrm{p}<0.001$, calculated by one-way analysis of variance test. 
tor cortex to the midbrain pons and medulla oblongata. It forms synapses directly or indirectly with the nuclei of facial, hypoglossal, and trigeminal cranial nerves to affect swallowing [19]. Therefore, activation of these nerves may affect swallowing muscles [19]. A study on the treatment of dysphagia by exciting the corticobulbar tract has reported positive results [20]. Therefore, lesions in the PLIC may cause dysphagia after MCA infarction and affect the response to recovery.

The swallowing mechanism can be affected by many neurons and muscles. Dysphagia can occur even when there is damage to only one of these. In this respect, it would be difficult to identify a single brain center associated with dysphagia. Therefore, among many lesions that can cause dysphagia, we think that the primary lesion most involved in the swallowing mechanism will become clearer after evaluating recovery from dysphagia rather than the incidence. A primary lesion with greater impact on recovery from dysphagia will be associated with longer duration of recovery regardless of the presence of absence of other lesions. However, classification of MCA infarction by lesion site has statistical limitations because independence between groups is not ensured as most lesions show overlap. Therefore, we evaluated lesions with longer mean recovery time without any difference in mean age, mRS score, or NIHSS score for all patients. We found that patients with PLIC and caudate nucleus lesions had longer mean recovery time compared to all patients (Table 2). In addition, the mean NIHSS score was lower for these lesions compared to that for other lesions while there was no significant difference in mean age, mRS score, or NIHSS score for all patients (Table 2). We classified groups according to overlap of these two lesions so that independence between groups was ensured (Table 3). As a result, patients with MCA infarction involving both the PLIC and caudate nucleus had significantly longer recovery time than patients without both lesions. There was no significant difference in recovery time for patients with a lesion in only one of these sites (Table 3).

In diffusion tensor imaging analysis of patients with MCA infarction, apparent diffusion coefficient value of the bilateral PLIC can differ significantly according to the presence or absence of dysphagia [11]. The caudate nucleus is associated with spatial working memory and directed movement $[21,22]$. Goal-oriented executive func- tioning is also closely associated with cognitive functions such as memory [23]. In contrast with the PLIC, the relationship between swallowing and the caudate nucleus is unclear. While further research is needed, it should be noted that these two regions receive similar blood supply [24].

We used the third stage of the dysphagia diet because it was the primary nutritional source for patients with cerebral infarction. These patients require $25 \mathrm{kcal} / \mathrm{kg}$ to prevent malnutrition [25]. Therefore, to provide adequate calories, a thin rice gruel diet is insufficient. The third stage diet contains $8 \mathrm{~g}$ of porridge and $10 \mathrm{~g}$ of thickener for a total of 1,765 kcal. This diet provides sufficient nutrition for rehabilitation therapy in patients with cerebral infarction. Therefore, a third stage diet can be the primary nutritional goal. In addition, unlike the thin rice gruel diet, the third stage diet requires sufficient recovery of oral and laryngeal phases. Therefore, the ability to tolerate the third stage diet is a sign that overall swallowing function has recovered.

Establishing a treatment plan for patients with dysphagia is an important part of rehabilitation therapy for stroke. Goal diet, determination of the proper diet for swallowing status, treatment method, duration of treatment, and more information can be determined with VFSS. However, VFSS may be delayed or omitted. Therefore, in the initial evaluation of patients with stroke, obtaining information related to dysphagia would help plan rehabilitation. In this study, the duration of dysphagia recovery increased with infarction of PLIC and caudate nucleus, providing important clues to the duration of recovery and the presence of dysphagia.

In patients with MCA infarction, the duration of dysphagia recovery was longer in patients with lesions of both the PLIC and caudate nucleus than that in patients without these lesions. Therefore, longer dysphagia treatment may be required for a patient with lesions of both the PLIC and caudate nucleus after an MCA infarction.

\section{CONFLICT OF INTEREST}

No potential conflict of interest relevant to this article was reported. 


\section{AUTHOR CONTRIBUTION}

Conceptualization: Kim JH, Kim GC. Methodology: Kim GC, Jeong HJ, Sim YJ. Formal analysis: Kim JH, Oh SH. Funding acquisition: none. Project administration: Kim GC, Jeong HJ. Visualization: Kim JH, Oh SH. Writing original draft: Kim JH, Oh SH. Writing - review and editing: all authors. Approval of final manuscript: all authors.

\section{REFERENCES}

1. Bray BD, Smith CJ, Cloud GC, Enderby P, James M, Paley L, et al. The association between delays in screening for and assessing dysphagia after acute stroke, and the risk of stroke-associated pneumonia. J Neurol Neurosurg Psychiatry 2017;88:25-30.

2. Martino R, Foley N, Bhogal S, Diamant N, Speechley M, Teasell R. Dysphagia after stroke: incidence, diagnosis, and pulmonary complications. Stroke 2005;36:2756-63.

3. Katzan IL, Cebul RD, Husak SH, Dawson NV, Baker DW. The effect of pneumonia on mortality among patients hospitalized for acute stroke. Neurology 2003;60:620-5.

4. Kelly J, Wright D, Wood J. Medicine administration errors in patients with dysphagia in secondary care: a multi-centre observational study. J Adv Nurs 2011;67:2615-27.

5. Mihai PG, Otto M, Domin M, Platz T, Hamdy S, Lotze $\mathrm{M}$. Brain imaging correlates of recovered swallowing after dysphagic stroke: a fMRI and DWI study. Neuroimage Clin 2016;12:1013-21.

6. Daniels SK, Pathak S, Mukhi SV, Stach CB, Morgan RO, Anderson JA. The relationship between lesion localization and dysphagia in acute stroke. Dysphagia 2017;32:777-84.

7. Suntrup S, Kemmling A, Warnecke T, Hamacher C, Oelenberg S, Niederstadt $\mathrm{T}$, et al. The impact of lesion location on dysphagia incidence, pattern and complications in acute stroke. Part 1: Dysphagia incidence, severity and aspiration. Eur J Neurol 2015;22:832-8.

8. Suntrup-Krueger S, Kemmling A, Warnecke T, Hamacher C, Oelenberg S, Niederstadt T, et al. The impact of lesion location on dysphagia incidence, pattern and complications in acute stroke. Part 2: Oropharyngeal residue, swallow and cough response, and pneumo- nia. Eur J Neurol 2017;24:867-74.

9. Fandler S, Gattringer T, Eppinger S, Doppelhofer K, Pinter D, Niederkorn K, et al. Frequency and predictors of dysphagia in patients with recent small subcortical infarcts. Stroke 2017;48:213-5.

10. Kim DS, Sim YJ, Kim CC, Jeong HJ. The relationship between swallowing disorder and physical function in stroke. Kosin Med J 2010;25:110-6.

11. Kim BR, Moon WJ, Kim H, Jung E, Lee J. Association of dysphagia with supratentorial lesions in patients with middle cerebral artery stroke. Ann Rehabil Med 2016;40:637-46.

12. Teismann IK, Dziewas R, Steinstraeter O, Pantev C. Time-dependent hemispheric shift of the cortical control of volitional swallowing. Hum Brain Mapp 2009;30:92-100.

13. Daniels SK, Foundas AL, Iglesia GC, Sullivan MA. Lesion site in unilateral stroke patients with dysphagia. J Stroke Cerebrovasc Dis 1996;6:30-4.

14. Robbins J, Levine RL, Maser A, Rosenbek JC, Kempster GB. Swallowing after unilateral stroke of the cerebral cortex. Arch Phys Med Rehabil 1993;74:1295-300.

15. Kern MK, Jaradeh S, Arndorfer RC, Shaker R. Cerebral cortical representation of reflexive and volitional swallowing in humans. Am J Physiol Gastrointest Liver Physiol 2001;280:G354-60.

16. Flowers HL, Skoretz SA, Streiner DL, Silver FL, Martino R. MRI-based neuroanatomical predictors of dysphagia after acute ischemic stroke: a systematic review and meta-analysis. Cerebrovasc Dis 2011;32:110.

17. Galovic M, Leisi N, Muller M, Weber J, Abela E, Kagi G, et al. Lesion location predicts transient and extended risk of aspiration after supratentorial ischemic stroke. Stroke 2013;44:2760-7.

18. Gonzalez-Fernandez M, Kleinman JT, Ky PK, Palmer JB, Hillis AE. Supratentorial regions of acute ischemia associated with clinically important swallowing disorders: a pilot study. Stroke 2008;39:3022-8.

19. Young PA, Young PH, Tolbert DL. Basic clinical neuroscience. 2nd ed. Philadelphia: Lippincott Williams \& Wilkins; 2007. p. 69-70.

20. Macrae PR, Jones RD, Huckabee ML. The effect of swallowing treatments on corticobulbar excitability: a review of transcranial magnetic stimulation induced motor evoked potentials. J Neurosci Methods 
2014;233:89-98.

21. Postle BR, D'Esposito M. Spatial working memory activity of the caudate nucleus is sensitive to frame of reference. Cogn Affect Behav Neurosci 2003;3:133-44.

22. Villablanca JR. Why do we have a caudate nucleus? Acta Neurobiol Exp (Wars) 2010;70:95-105.

23. Grahn JA, Parkinson JA, Owen AM. The role of the basal ganglia in learning and memory: neuropsycho- logical studies. Behav Brain Res 2009;199:53-60.

24. Kurabe S, Okamoto K, Suzuki K, Matsuzawa H, Watanabe M, Suzuki Y, et al. The posterior limb of the internal capsule as the subcortical transitional zone of the anterior and posterior circulations: insights from human 7T MRI. Cerebrovasc Dis 2016;41:256-64.

25. Bouziana SD, Tziomalos K. Malnutrition in patients with acute stroke. J Nutr Metab 2011;2011:167898. 